\title{
Review
}

\section{Research Progress on HIV/AIDS with Concomitant Hepatitis B Virus and/or Hepatitis C Virus Infection}

\author{
Fuying Guo, Lingzhou Yang \\ Infection Management Section, The People's Hospital of Lincang, Lincang, China
}

\author{
Keywords \\ HIV; AIDS; HBV; HCV; Mixed infection; Super \\ infection
}

\section{Correspondence}

Lingzhou Yang, E-mail: Icyyylz@163.com

DOI: $10.1515 / i-2017-0099$

\begin{abstract}
Hepatitis B virus (HBV), hepatitis C virus (HCV), and human immunodeficiency virus (HIV) involve similar transmission routes, namely, blood, sexual contact, and mother-baby contact. Therefore, HIV infection is usually accompanied by HBV and HCV infections. This observation poses a great challenge to the prevention and treatment of HIV/human acquired immunodeficiency syndrome (AIDS) accompanied by HBV and HCV infection. Highly active antiretroviral therapy (HAART) has been extensively applied. Hence, liverrelated diseases have become the main causes of complication and death in HIV-infected individuals. This paper summarizes the current epidemiology, mutual influence, and treatment of HIV/AIDS accompanied by HBV or HCV infection.
\end{abstract}

More than 20 years have passed since the human immunodeficiency virus (HIV) was first reported in the United States. Since then, HIV has gradually become a highly serious public health problem of global concern. Chronic Hepatitis B virus (HBV) and chronic hepatitis C virus $(\mathrm{HCV})$ are chronic hepatitis viruses that jeopardize human health for a long period. HIV, HBV, and HCV involve similar transmission routes, namely, blood, sexual contact, and mother-baby contact. Hence, concurrent infection (mixed infection/superinfection) by these three viruses is possible. In recent years, with the increasing number of people engaged in intravenous drug use and sexual openness, the number of people infected with HIV and HBV/HCV gradually increased each year. Highly active antiretroviral therapy (HAART) have been extensively promoted, and the death rate in HIV/AIDS and incidence rate of opportunistic infection and other complications have gradually decreased. Hence, the development of chronic liver diseases in patients infected with HIV/AIDS and HBV/HCV has become a main cause of death in HIV patients. Therefore, concomitant HIV and $\mathrm{HBV} / \mathrm{HCV}$ infections have gained increasing attention.

\section{Prevalence}

\section{Worldwide}

At present, more than 300 million HBsAg carriers exist in the world ${ }^{[1]}$. This number accounts for about $5 \%$ of the total population. The incidence rate of mixed HIV/AIDS and
HBV infection differs significantly among different regions and different high-risk groups. In the regions of Europe and America with low prevalence of hepatitis $\mathrm{B}$, the $\mathrm{HBV}$ infection rate in HIV-infected individuals is 6\%-10\%. This percentage is higher than the infection rate in normal people. In Asia and Africa, the HBV infection rate in HIV-infected patients is similar to or even lower than the infection rate in normal people ${ }^{[2]}$. In American people with HIV infection, the $\mathrm{HBV}$ infection rate is $7 \%-10 \%$. In male homosexuals with $\mathrm{HIV}$ infection, the HBsAg positive rate is as high as $10 \%^{[3]}$. Meanwhile, the repeated use of blood products is another strong risk factor related to mixed $\mathrm{HIV} / \mathrm{HBV}$ infection. In a foreign survey, $63.7 \%$ of 160 hemophilia patients who received multiple blood transfusions presented with mixed $\mathrm{HIV} / \mathrm{HBV}$ infection ${ }^{[4]}$. About 180 million people are infected by HCV worldwide ${ }^{[5]}$; this number accounts for $2.8 \%$ of the total population. The HCV infection rate in HIV/ AIDS patients is as high as $25 \%$, and this rate changes with infection route. Intravenous drug use is an important cause of mixed HIV/HCV infection. Of the patients infected by HIV through intravenous drug use, 70\%-90\% also presented with $\mathrm{HCV}$ infection ${ }^{[6]}$. The use of contaminated blood and blood products is another strong risk factor for mixed HIV/HCV infection. Dragoni et al. reported that the HIV infection rate in hemophilia patients is $32.3 \%$, and the $\mathrm{HCV}$ infection rate reaches $88.2 \%$. However, according to various reports, sexual contact causes mixed HIV/HCV infection at low probability. In male homosexual patients with HIV infection, the 
proportion with concurrent $\mathrm{HCV}$ infection is similar to that in HIV-negative patients, and the infection rate is $4 \%-8 \%[7,8]$. The incidence rate of mixed infection caused by heterosexual contact is lower. Pineda et al. ${ }^{[9]}$ reported that among 294 prostitutes with no intravenous drug use in Spain, 6\% suffer from mixed HIV/HCV infection. Research on mother-baby transmission showed that in the patients with concurrent infection, the vertical transmission rate of $\mathrm{HIV} / \mathrm{HCV}$ was higher. No large-scale clinical epidemiological data on the concomitant infection of the three viruses have been reported.

\section{China}

Since the first HIV patient was confirmed in China in 1985, HIV-infected individuals and AIDS patients have been constantly found. According to the introduction to the AIDS Prevention and Care Conference of the Red Cross Society of China held in November 2010, nearly 3.2 million AIDS patients and HIV-infected individuals were cumulatively reported in China by October 31, 2009. This number included more than 100000 AIDS patients. By the end of 2009, a total of 740000 living AIDS patients and $\mathrm{HIV}$-infected individuals reside in China. As regards mixed HIV/HBV infection, information from a largescale epidemiological survey is needed in China. The local study results are basically consistent with foreign data. In China, the main reason for mixed HIV/HBV infection is intravenous drug use. Among the patients with history of intravenous drug use, HBV and HIV were recorded with the same transmission rates. In the patients infected by HIV through intravenous drug use, the $\mathrm{HBV}$ infection rate is as high as $93.6 \%{ }^{[10]}$. In the rural area, the infection rate related to paid blood donation is $2.0 \%-8.2 \%^{[11]}$. For mixed HIV/ $\mathrm{HCV}$ infection, the main transmission routes in China are intravenous drug use and blood transfusion products, which are consistent with those reported globally. According to a report, the incidence rate of mixed $\mathrm{HIV} / \mathrm{HCV}$ infection through intravenous drug use is $51 \%^{[12]}$. Moreover, the incidence rate of mixed HIV/HCV infection caused by blood transfusion is $44 \%^{[12]}$. Finally, the incidence rate of mixed $\mathrm{HCV}$ infection in HIV-1 positive patients derived from blood transmission is $94 \%^{[12]}$.

\section{Mutual influence among different viruses in concurrent infection}

\section{Mutual influence between HBV and HIV}

In gaining natural immunity against $\mathrm{HBV}$ infection, $\mathrm{CD} 4^{+}$
$\mathrm{T}$ cells play an important role. When the human body is infected with $\mathrm{HBV}$, Th1 $\mathrm{CD}^{+} \mathrm{T}$ cells promote the activation and proliferation of cytotoxic T CTL, natural killer cells (NK cells), and macrophagocytes by secreting (IL)-2 and interferon $\gamma($ IFN- $\gamma)$. Meanwhile, the up-regulation of IFN- $\gamma$ induces the body to produce nitric oxide synthase (NOS), which inhibits $\mathrm{HBV}^{[13]}$. In a body with mixed HIV/HBV infection, HIV damages $\mathrm{CD}^{+}{ }^{+} \mathrm{T}$ cells in various ways and reduces the cells' ability to remove the $\mathrm{HBV}$. As a result, the body tolerates and retains the HBV. Therefore, HBV chronicity and continuous viremia ensue in the body with concurrent infection. A French research proposed that severe liver diseases caused by viral hepatitis have become the main reason for death in HIV/AIDS patients. The death rate in $\mathrm{HIV} / \mathrm{HBV}$ double infection cases due to severe liver diseases is significantly higher than that of the regular population ${ }^{[14]}$. Furthermore, during HBV duplication in the body, the encoded HBX protein of the $\mathrm{X}$ protein code area of the long negative chain widely activates viral and cellular promoters. Consequently, the expression of multiple types of tumor cells is activated. The mechanism by which chronic HBV infection causes primary hepatocellular carcinoma is a research hotspot. Some studies showed that during HBV/HIV infection, $\mathrm{HBX}$ influences not only $\mathrm{HBV}$ progression but also HIV progression. At the two ends of the HIV genome, a long LTR exists. This LTR mainly functions for the expression and adjustment of virogenes. Meanwhile, the HIV Tat effect elements are distributed on LTR. Tat combines with the RNA transcribed from the area and then improves the HIV gene transcription level. Existing research has proven that HBX promotes HIV activation, transcription, and duplication in the body through its own transactivation. Therefore, in a body under mixed HIV/HBV infection, HBV also accelerates HIV progression. In recent years, in-depth research on relevant mechanisms have indicated that the HBX protein induces HIV-1 duplication and HIV-1LTR transcription through synergistic effects of the Tat protein and T-cell activation of signal pathways.

\section{Mutual influence between HIV and HCV \\ Influence of HIV on HCV}

The incidence rate of mixed $\mathrm{HIV} / \mathrm{HBV}$ infection is significantly higher than that of mixed HIV/HBV infection. Thus, scholars have conducted numerous related clinical observational studies. A case-control study reported that the time from $\mathrm{HCV}$ infection to liver cirrhosis was significantly shorter in HIV/HCV-infected patients than in HCV-only patients $^{[15]}$. In $10-15$ years, $15 \%-25 \%$ of $\mathrm{HIV} / \mathrm{HCV}$ infections 
developed to liver cirrhosis. Within the same period, only 2\%-6\% of HIV infections and HIV-negative cases developed to liver cirrhosis. A British research on hemophilia found that the death rate of individuals super infected with HIV/ $\mathrm{HCV}$ who progressed to liver-related diseases was 4-6 times that of those infected with HIV alone ${ }^{[16]}$. Abundant clinical observational studies have proven that, with respect to $\mathrm{HCV}$ infection alone, concurrent HIV infection accelerates HCV infection, improves HCV duplication, worsens HCV attacks to the liver, and accelerates liver cirrhosis progression. At present, the specific mechanism of action of HCV in the body is unclear. However, most studies proposed that such mechanism is closely related to the CTL function. The main function of CTLs in viral immunity is identifying the provirus compound of the APC surface and MHC-1 molecules through the TCR on its surface and killing infected target cells. Meanwhile, secret able cytokines are involved in killing and immune adjustment. Secreted cytokines mainly include IFN- $\gamma$, tumor necrosis factor a (TNF- $\alpha$ ), interleukin (IL)-8, granulocyte-macrophage colony-stimulating factor (GM-CSF), and IL-10. Rainer et al. ${ }^{[17]}$ found that the IL-2 and IL-10 production in the body of a patient with mixed HIV/HCV infection is significantly increased. IL-10 involves an immunosuppressive effect. This information implies that HIV obviously changes the specific cytokine reaction of $\mathrm{HCV}$, and this alteration may accelerate $\mathrm{HCV}$ progression in mixed infection. Furthermore, HIV infection causes the $\mathrm{CD}^{+} \mathrm{T}$ cells to reduce the cell immunity of the body against $\mathrm{HCV}$ in the acute stage. This occurrence results in persistent $\mathrm{HCV}$ infection. HIV-1variation induces HIV-specific CTLs to enter a state of unresponsiveness and causes autoimmune disorder. These effects eventually lead to HCV survival and expansion. All of these mechanisms would likely result in chronic HCV progression in mixed HIV/ $\mathrm{HCV}$ infection. However, the specific mechanism requires further research.

\section{Influence of HCV on HIV}

In foreign and domestic research, different opinions exist on whether HCV infection influences HIV progression. Some foreign scholars conducted a 6-year cohort study on 1995 patients with mixed HIV/HBV infection. No increase in AIDS incidence rate or AIDS-related death rate was found ${ }^{[18]}$. After HIV treatment was adjusted, HCV was not independently associated with death in AIDS patients with $\mathrm{CD}^{+} \mathrm{T}$ cell count of $50-200 / \mu \mathrm{L}$. Chinese scholars Luo Jiala et al. ${ }^{[19]}$ believed that HIV progression is not closely related to $\mathrm{HCV}$ infection. However, many scholars insist that the increased AIDS-related death risk and increased possibility of developing AIDS in HIV-infected patients are related to $\mathrm{HCV}$ infection in an unknown mechanism. Some believe that this effect is caused by the inactivation of $\mathrm{CD}^{+}{ }^{+} \mathrm{T}$ cell reaction after HCV serum-positive patients receive HAART ${ }^{[20]}$. Some works reported that mixed HIV/ $\mathrm{HCV}$ infection quickly reduces the number of CD4+ T lymphocyte in HIV-infected patients and accelerates HIV progression $^{[21]}$. However, the effect of mixed HIV/HCV infection on the case fatality rate remains controversial.

\section{Treatment of concurrent infection}

\section{Treatment of $\mathrm{HIV} / \mathrm{HBV}$ mixed infection}

Through time, the number of patients with HIV/HBV mixed infection has continuously increased; HAART treatment has been promoted; and the HIV/HBV death rate, opportunistic infection, and other complications have gradually decreased. Concomitantly, chronic liver diseases have become the major causes of death of patients infected with HIV. Therefore, the treatment of $\mathrm{HIV} / \mathrm{HBV}$ mixed infection is attracting increasing attention. At present, a unified treatment goal has been achieved. This goal includes the blocking and delay of disease progression, reduction of the incidence rate of endstage liver diseases, decrease in HAAR The pat toxicity, and lowering of the risk of immune reconstitution syndrome ${ }^{[22]}$. However, no consensus has been reached on the selection of specific treatment opportunity and drugs. As regards the selection of treatment opportunity, the American Association for the Study of Liver Diseases pointed out in the Chronic Hepatitis B Guidelines ${ }^{[23]}$ in 2006 that anti-HBV treatment should be adopted for patients with mixed HIV/ HBV infection who satisfied the chronic HBV criteria. The guidelines further stated that the use of anti-HIV treatment on patients with mixed HIV/HBV infection could reduce liver disease progression and lower the incidence rate of drugresistant mutation ${ }^{[24]}$. In terms of drug selection, various foreign and domestic chronic hepatitis Guidelines may serve as bases. Tenofovir disoproxil (TDF) plus emtricitabine and a third anti-HIV drug are recommended for HAART. Currently, clinical research on various drugs mainly focuses on TDF. In a prospective double-blind placebo study, 52 patients with mixed HIV/HBV infection who were subjected to HAART randomly used Adefovir dipivoxil (ADV) 10 $\mathrm{mg} /$ day and TDF $300 \mathrm{mg} /$ day. At the baseline, the amount of HIV-1DNA of $73 \%$ of the patients was lower than 50 copy/ $\mathrm{ml}$. Then, $86 \%$ of the patients were $\mathrm{HBeAg}$ positive, and $94 \%$ of the patients showed LAM drug-resistant mutation. After the treatment was applied for 48 weeks, the amount 
of HBV-DNA was reduced by $4.44 \log _{10}$ copy/mL (TDF) and $3.21 \log _{10} \mathrm{copy} / \mathrm{ml}(\mathrm{ADV})^{[25]}$, respectively. Likewise, some retrospective studies also showed TDF to significantly reduce the HBV-DNA level of patients with mixed HIV/HBV infection. However, no sufficient large-scale clinical research data are available on emtricitabine.

\section{Treatment of HIV/HCV mixed infection}

The HCV treatment goalsare to reduce blood plasma HCVRNA levels to below test levels at the therapeutic endpoint,to maintain an inhibitory effect for at least 6 months after drug withdrawal, and to achieve a persistent viral response ${ }^{[26]}$. Concurrent HIV/HCV infection accelerates HCV progression, and HAART may alter the balance between the body's immune system and HCV. As a result, immunopathologic damage is produced. Therefore, effective anti-HCV treatment minimizes the threat of end-stage liver diseases in HIV/HCV patients. However, the ideal treatment opportunity, optimal therapeutic regimen, and treatment course in concurrent infection is unclear. At present, most scholars agree on the following standard therapeutic regimen: PEG-IFN and ribavirin used in combination for 48 weeks. For genotypes 2 and 3, ribavirin $800 \mathrm{mg}$ /day should be added. For genotypes 1 and 4, 1000-2000 mg/day should be used. In a study by Torriani et al. ${ }^{[27]}$, each of the three regimens was randomly given to 868 patients. As a result, the sustained virologic response rates in the HIV/HCV patients achieved for the common IFN $\alpha-2 \mathrm{a}$ ( 3 million $\mathrm{U}$, twice/week) and ribavirin ( $800 \mathrm{mg} /$ day), PEG-IFNa-2a $(180 \mu \mathrm{g} /$ week $)$ and placebo, and PEG-IFN $\alpha-2 \mathrm{a}(180 \mu \mathrm{g} /$ week) and ribavirin ( $800 \mathrm{mg} /$ day) were $12 \%, 20 \%$ and $40 \%$, respectively. In genotype 1 , theSVR for PEG-IFN $\alpha$-2a and ribavirin was $29 \%$. In genotypes 2 and 3 , the SVR was $62 \%$. Meanwhile, regimen 3 achieved the highest histologic liver improvement (57\%), which was much higher than the $39 \%$ of regimen 1 and $41 \%$ of regimen 2 .

\section{Treatment of HIV infection accompanied by $\mathrm{HBV}$ and HCV infections}

Most studies on concomitant HIV and HBV or HCV infection are still in the stage of initial exploration. Current research focuses on whether the mixed infection influences the HAART effect. Cooper et al. ${ }^{[28]}$ adopted regular HAART for 38 patients infected with HBV, HCV, and HIV. The group found that mixed virus infection did not influence the HAART effect. However, a research by Martin-Carbonero et al. ${ }^{[29]}$ demonstrated that negative or positive HCV-RNA influences liver disease progression and the choice of
HAART regimen. All the above-mentioned studies were based on the reasonable selection of HAART regimen. Li Xiaofei et al. ${ }^{[30]}$ adopted HAART (zidovudine + didanosine + nevirapine) for patients infected with all three viruses (HIV, $\mathrm{HBV}$, and HCV). The scholars observed that all the groups achieved rapid HIV-RNA inhibition, accompanied with increased $\mathrm{CD} 4^{+} \mathrm{T}$ lymphocyte count at varying degrees. The findings showed that $\mathrm{HBV}$ and $\mathrm{HCV}$ infection exerted no obvious influence. The treatment effect of HAART requires further research and analyses.

\section{Prevention of HIV infection with HBV and/or HCV coinfection}

At present, no effective vaccine is available for HIV or HCV. Their main transmission routes are the blood, sexual contact, and mother-baby contact and are hence called behavioral diseases. Behavioral intervention should be mainly observed to prevent concurrent infection. Specific measures include control of intravenous drug use, syringe exchange, methadone replacement therapy, blood resource management reinforcement, and safe sex education. Meanwhile, liver disease progression could be delayed and the patients' quality of life could be improved by several measures. Examples of such strategies include popularization of health knowledge, routine screening, early detection of patients with concurrent infection, and timely provision of active treatment. In addition, enhancing vaccine development is the most fundamental and most effective method to prevent infection, especially mixed HIV/HCV infection.

\section{Declarations}

\section{Acknowledgements}

No.

\section{Competing interests}

The authors declare that they have no competing interest.

\section{Authors' contributions}

FY Guo made the literature analysis and wrote, discussed and revised the manuscript of this review. LZ Yang critically analyzed and corrected the manuscript. All authors read and approved the final manuscript.

\section{References}

1 Meng C, Yang L, Wang J, et al. Significance of cellular immune function 
for HBV virus replication and antiviral treatment. Zhongguo Quan Ke Yi Xue, 2010,13(7): 2226.

2 Christopher JH, Chloe LT. Clinical implications of HIV and hepatitis B co-infection in Asia and Africa . Lancet Infect Dis, 2007, 7(6): 402-409.

3 Soriano V, Puoti M, Bonacini M, et al. Care of patients with chronic hepatitis B and HIV co-infection: recommendations from an HIV/HBV international panel. AIDS, 2005, 19(3): 221-240.

4 Saillour F, Dabis F, Dupon M, et al. Prevalence and determ in ants of antibodies to hepatitis $\mathrm{C}$ virus and markers for hepatitis $\mathrm{B}$ virus in patients with HIV infection in Aquitaine. BMJ, 1996, 313(7055): 461-464.

5 Thio AL, Seaberg EC, Skolasky RL, et al. HIV-1, hepatitis B virus, and risk of liver-related mortality in the multi center AIDS cohort study. Lancet, 2002, 360 (9349): 1921-1926.

6 Hagan $\mathrm{H}$, Thiede $\mathrm{H}$. Sharing of drug preparation equipment as a risk factor for hepatitis C. Am J Public Health, 2001, 91: 42-46.

7 Dragoni F, Gentile G, Cartoni C, et al. The role of realtime ultrasonography in predicting esophageal varices in hemophiliacs co-infected with hepatitis $\mathrm{C}$ and human immunodeficiency virus. Haematology, 2005, 90(2): 207-213.

$8 \mathrm{Hu}$ Y. Progress of research on mixed infection of HIV and HCV. Zhongguo Ai Zi Bing Xing Bing, 2005, 11(3): 233-234.

9 Pineda JA, River A, Rey C, et al. Association between hepatitis C virus seroreactivity and HIV infection in non-intravenous drug abusing prostitutes. Clin Microbiol Infect Dis, 1995, 14(5): 460-464.

10 Wei D, Ma M. A survey on HIV/HBV/HCV infection among drug users. Yu Fang Yi Xue Qing Bao Za Zhi, 2000, 16(2): 187-188.

11 Dong P, Li Z, Zhang K, et al. An analysis on HCV/HBV infection of 44 HIV/AIDS patients. Zhongguo Gong Gong Wei Sheng, 2003, 19(7): 866.

12 Cao Y. Outlook for HIV clinical epidemiology and prevention and control. Guo Wai Yi Xue Liu Xing Bing Xue Chuan Ran Bing Fen Ce, 2002, 29(1): 4 .

13 Rehermann B, Nascmen IM. Immunology of hepatitis B virus and hepatitis C virus infection. Nat Rev Immunology, 2005, 5(3): 215-229.

14 Lewden C, Salmon D, Morlat P, et al. Causes of death among human immunodeficiency virus ( HIV)-infected adults in the era of potent antiretroviral therapy emerging role of hepatitis and cancers persistent role of AIDS. Int J Epidemiology, 2005, 34(1): 121-130.

15 Jurgen KR, Ulrich S. HIV and hepatitis C virus co-infection . Lancet Infect, 2004, 4(7): 437-444.
16 Mohsen AH, Easterbrook P, Toylor CB, et al. Hepatitis C and HIV coinfection. Gut, 2002, 51(4): 601-608.

17 Rainer PJ, Urgen KR, Imke B, et al. Antigen-specific cytokine respond to hepatitis $\mathrm{C}$ virus core epitopes in HIV/hepatitis C viruscon-infected patients. AIDS, 1999, 13(11): 1313-1322.

18 Sulkowski MS, Moore RD, Mehta SH, et al. Hepatitis C and progression of HIV disease. JAMA, 2002, 288(2): 199-206.

19 Luo J, Gui X, Zhuang K. HCV/HBV infection among HIV patients. Shijie Hua Ren Xiao Hua Za Zhi, 2003, 11(11): 1835-1836.

20 Greb G, Lederger B, Battegay M, et al. Clinical progression survival and immune recovery during antiretroviral therapy in patients with HIV-1 and hepatitis C virus coinfection: the Swiss HIV cohort study. Lancet, 2000, 356(9244): 1800-1805.

21 Dong $\mathrm{P}$, Zhang K, Wu H, et al. Mutual influence of HIV virus and HCV virus infection of 140 adults. Lin Chuang Hui Cui, 2004, 19(2): 61-63.

22 Crockett SD, Keeffe EB. Natural history and treatment of hepatitis B virus and hepatitis $C$ virus coinfection. Ann Clin Microbiol, 2005, 4: 13.

23 Lok AS, McMabon BJ. Chronic hepatitis B. Hepatology, 2007, 45(2): 507-539.

24 Hammer MG, Eron J, Reiss P, et al. Antiretroviraltreatment of adult HIV infection: 2008 recommendations of the international AIDS societyUSA panel. JAMA, 2008, 300(5): 555-570.

25 Peters MG, Andersen J, Lynch P, et al. Randomized controlled study of tenofovir and adefovirin chronic hepatitis B virus and HIV infection. Hepatology, 2006, 44(5): 1110-1116.

26 Albert A, Clumeck N, Collins S, et al. Short statement of the first European consensus conference on the treatment of chronic hepatitis B and C in HIV co-infected patients. Hepatology, 2005, 2(5): 615-624.

27 Torriani FJ, Rodriguez-Torres M, Rockstroh JK, et al. Peg-interferon Alfa plus ribavirin for chromic hepatitis $\mathrm{C}$ virus infection in HIV-infected patients. N Engl J Med, 2004, 351(5): 438-450.

28 Cooper CL, Mills E. Comparison of first antiretroviral treatment duration and outcome in HIV, HIV-HBV and HIV-HCV infection. Int J STD AIDS, 2007, 18(8): 546-550.

29 Martin-Carbonero L, Barreiro P, Jimenez-Galam G, et al. Clearance of hepatitis Cvirus in HIV-infected patients with multiplechronic virus hepatitis. J Viral Hepat, 2007, 14(6): 392-395.

30 Li X, Kan Q He Y, et al. Influence of mixed infection of HIV, HCV and HBV on HAART effect. Zhonghua Nei Ke Za Zhi, 2010, 49(11): 951-954. 